\title{
STRAIN SENSING WITH PIEZOELECTRIC ZINC OXIDE THIN FILMS FOR VIBRATION SUPPRESSION IN HARD DISK DRIVES
}

\author{
Sarah Felix \\ Department of Mechanical Engineering \\ University of California \\ Berkeley, California 94720 \\ Email: sarfelix@me.berkeley.edu
}

\author{
Stanley Kon \\ Jianbin Nie \\ Roberto Horowitz \\ Department of Mechanical Engineering \\ University of California \\ Berkeley, California 94720 \\ Email: horowitz@me.berkeley.edu
}

\begin{abstract}
This paper describes the integration of thin film $\mathrm{ZnO}$ strain sensors onto hard disk drive suspensions for improved vibration suppression for tracking control. Sensor location was designed using an efficient optimization methodology based on linear quadratic gaussian ( $L Q G)$ control. Sensors were fabricated directly onto steel wafers that were subsequently made into instrumented suspensions. Prototype instrumented suspensions were installed into commercial hard drives and tested. For the first time, a sensing signal was successfully obtained while the suspension was flying on a disk as in normal drive operation. Preliminary models were identified from experimental transfer functions. Nominal $\mathrm{H}_{2}$ control simulations demonstrated improved vibration suppression as a result of both the better resolution and higher sensing rate provided by the sensors.
\end{abstract}

\section{INTRODUCTION}

In a conventional hard disk drive, illustrated in Fig. 1, head positioning is achieved by operating a voice coil motor (VCM) with feedback control. Special bits, called servo tracks, are permanently written onto the disk along with the data bits. As the magnetic head reads data on the disk, it also periodically reads the servo tracks, obtaining a position error signal (PES). The PES is fed back to the VCM to provide servo control for positioning the head. As areal data density in hard disk drives continues to increase, hard drives require much better head positioning accuracy. The track mis-registration (TMR) budget for the current industry goal of 1 terabit per square inch requires positioning accuracy within $5 \mathrm{~nm}$ at $3 \sigma$ root-mean-square (RMS) tracking

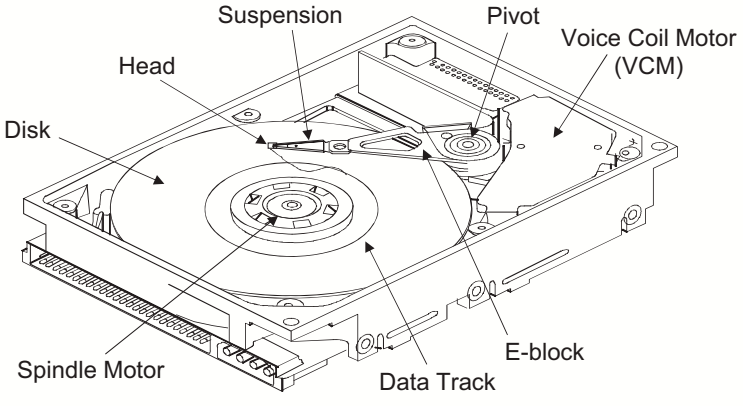

Figure 1. A CONVENTIONAL HARD DISK DRIVE.

error. High frequency airflow-induced vibrations pose a major barrier for conventional disk drive servos to meet these requirements, necessitating new sensing and actuation strategies.

The bandwidth of conventional drives is limited for two main reasons. First, the frequency of the PES signal is constrained by the number of servo sectors on the disk and the rotation rate. This prevents the servo from compensating for higher frequency disturbances. Adding more servo sectors would decrease the amount of disk space available for data bits. Second, vibration modes along the suspension, downstream of the VCM, make nanometer-scale positioning at the head difficult. Several researchers have proposed dual stage servo configurations in which a second actuator is placed on the suspension or near the head for higher-frequency, micro-scale positioning [1-3]. However, such schemes may require high-rate vibration sensing that 
cannot be obtained from the PES. It is clear that a strain sensor measuring vibration at a higher rate would facilitate control of the head position.

Previously, Li implemented strain sensing along with dual stage control using suspensions fabricated with two lead zirconate titanate (PZT) strips near the suspension hinge [4]. One PZT strip was used for sensing and the other for actuation. While this scheme did improve tracking performance, there were several drawbacks. The bulk PZT transducer significantly altered the dynamics of the suspension, and the strain signal was not optimal for tracking control. It is desirable to place sensors so that important off-track vibration information is detected, while signals from non-off-track modes are minimized. A standard linear quadratic gaussian (LQG) cost function has been used to incorporate controller structure and to weight the signals from desired modes [5]. The solution to this optimization is computationally intensive, making it difficult to evaluate many sensor configurations. Oldham et al. introduced an algebraic approximation to the LQG-based optimization that has been applied to strain sensor design for disk drive suspensions [6].

Strain sensing using thin films provides an opportunity to monitor strain on a disk drive suspension without significantly altering the dynamics of the suspension structure. Piezoelectric films, in particular, are suitable for high-frequency dynamic sensing because the material's capacitive response tends to act as a high-pass filter, rejecting lower frequency noise and amplifying the signal of interest. Kon demonstrated successful fabrication and operation of thin film $\mathrm{ZnO}$ strain sensors on stand-alone instrumented suspensions [7].

This paper demonstrates implementation of instrumented suspensions in an operating disk drive. We also investigated improvements to vibration suppression using instrumented suspensions with $\mathrm{ZnO}$ strain gages. A model was identified from open loop experimental results. Then, equipped with this realistic model, nominal $\mathrm{H}_{2}$ control synthesis was used to evaluate closed-loop vibration suppression. Results revealed the effectiveness of high-rate strain sensing for off-track positioning. The organization is as follows: The next section will review the sensor design method; the third section will discuss fabrication and material characterization; the fourth section describes the experimental testing and results; the fifth section discusses modeling and control simulations; the final section provides conclusions and future work.

\section{SENSOR DESIGN Optimization Algorithm}

For disk drive servo control, the most detrimental vibration modes are those causing motion perpendicular to the data track. Thus, a cost function for optimizing sensor location should weight more heavily vibration signals from such modes. A quadratic cost function based on LQG control [8] can be constructed which penalizes off-track error under closed-loop control using estimations from sensor signals. Thus, the cost function directly evaluates how effective a sensor configuration would be in a closed-loop controller. A state space model of the disk drive servo system can be constructed as follows:

$$
\begin{aligned}
& \dot{x}=A x+B u+B_{w} w \\
& y=C(\Phi) x+v(\Phi) \\
& z=D x,
\end{aligned}
$$

where $x$ is the state vector, $A, B, B_{w}, C$, and $D$ are system matrices, $u$ is the actuator input, $w$ is the windage input disturbance with spectral density $W, v$ is the measurement noise with spectral density $V, y$ is the sensor strain measurement, and $z$ is the off track error. $\Phi$ refers to the specific sensor configuration. An LQG controller minimizes the following $H_{2}$ norm, $J_{H_{2}}$ :

$$
J_{H_{2}}=\min _{K, F(\Phi)} E\left[z^{T} z+u^{T} R u\right]
$$

where $F(\Phi)$ denotes the Kalman filter gain [8] for a given $C(\Phi)$, and $K$ denotes the optimal linear stationary controller [8]. The variance of off track error, $z$, is penalized, and $R$ is a matrix that penalizes the actuator effort. However, since the actuator range of motion is typically much larger than the suspension vibrations, the problem may be simplified by solving the case of "cheap control", i.e. $R \rightarrow 0$, as described in [6]. Note that the norm $J_{H_{2}}$ is a function of sensor configuration, $\Phi$, so the sensor optimization problem is stated as

$$
\min _{\Phi} J_{H_{2}}(\Phi)
$$

The computation of $J_{\mathrm{H}_{2}}$ requires the solution of a Riccati equation, making the evaluation of many sensor configurations numerically intensive. However, two conditions that tend to be satisfied in hard drive suspensions lead to an algebraic approximation of the solution [6].

1. Vibration modes are widely spaced:

$$
\left|\omega_{i}-\omega_{j}\right|>>0
$$

where $\omega_{i}$ is the resonant frequency of mode $i$.

2. Sensor noise is large relative to other parameters:

$$
\left|c_{j i}\right|\left(\sum_{k=1}^{l}\left|b_{i} k\right|\right) \sqrt{\frac{\mathrm{w}}{\mathrm{v}}}<<1,
$$

for all $i$ and $j$, where $l$ is the number of states.

Then, the quadratic cost function can be approximated by

$$
J_{H_{2}} \approx \sum_{j=1}^{N} \frac{d_{j}^{2} b_{j} \sqrt{\mathrm{WV}}}{\sqrt{c_{1 j}^{2}+\ldots+c_{r j}^{2}}}
$$


This expression significantly reduces computation time and provides better intuition about how different system parameters enter into the cost. See [6] for details on the validity of the above assumptions and the derivation of the approximation in Eq. (6).

\section{Implementation on Instrumented Suspensions}

To implement this optimization method on instrumented suspensions, a finite element model of the suspension was built to obtain $x-, y-$, and shear components of strain. The strain was computed at each element of the spatially-discretized model according to contributions from each mode. For example, the matrix

$$
C_{\varepsilon_{x}}(p)=\left[\begin{array}{llllll}
c_{x 1} & 0 & c_{x 2} & 0 & \ldots & 0
\end{array}\right]
$$

describes the contribution of each vibration mode to strain, $\varepsilon_{x}$, in the $x$-direction at element $p$. Similar vectors are calculated for strain in the $y$ - and shear $(x y-)$ directions. Then the $C(\Phi)$ matrix is given by

$$
C(\Phi)=\frac{\sum_{p \in \Phi} A_{p} K_{s}\left[\begin{array}{c}
C_{\varepsilon_{x}}(p) \\
C_{\varepsilon_{y}}(p) \\
C_{\varepsilon_{x y}}(p)
\end{array}\right]}{C_{p}+\sum_{p \in \Phi} \frac{A_{p} \varepsilon_{p}}{t}} .
$$

In this expression, $A_{p}$ is the area of element $p, K_{s}$ is a matrix of piezoelectric coefficients in the $x-, y-$, and shear directions, $C_{p}$ is the parasitic capacitance of external leads, $\varepsilon_{p}$ is the permissivity of the piezoelectric film, and $t$ is the strain gage thickness. This $C(\Phi)$ matrix is computed for every sensor configuration and the cost function is evaluated using the algebraic approximation in Eq. 6. Figure 2 shows an ANSYS model of one sensor configuration near the hinge of a suspension. Note that the ANSYS model need only be run once to obtain coefficients for each element. A program was written to automatically generate reasonable combinations of elements and compute their corresponding cost function. In this way, more than fifty sensor configurations were easily evaluated.

\section{Cancelation of non-off-track modes}

Even an optimized sensor configuration inevitably captures some non-off-track displacement signals. This could lead to overuse of the actuator as it tries to compensate for these modes. However, it is possible to exploit symmetry in a sensor configuration to minimize the response from non-off-track modes. If two symmetrical sensors are placed on each side of the suspension hinge, they will pick up equal signals from non-off-track displacement modes, such as the first bending mode. On the other hand, signals from off-track modes will be 180 degrees out of phase. Thus, if the signal from one sensor is subtracted from the other, off-track signals will be amplified by a factor of two, while

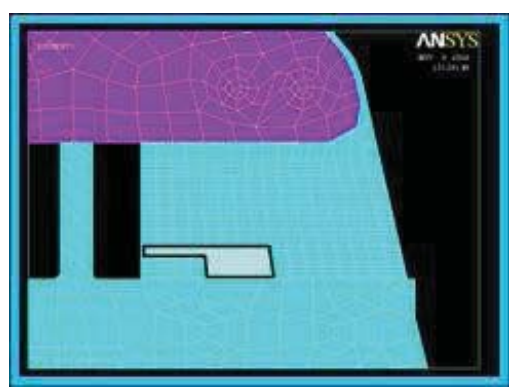

Figure 2. ANSYS MODEL OF ONE SENSOR CONFIGURATION NEAR THE HINGE OF AN INSTRUMENTED SUSPENSION.

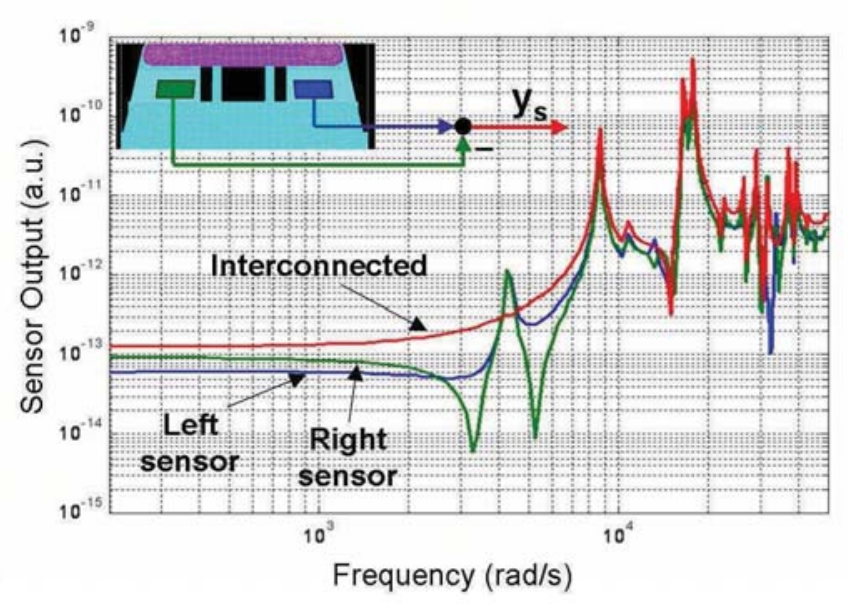

Figure 3. USING SYMMETRY TO CANCEL NON-OFF-TRACK MODES.

non-off-track modes will be canceled. Figure 3 illustrates this in a simple simulation. For this reason, the instrumented suspension prototypes were designed to have identical sensors on each side of the suspension hinge.

\section{FABRICATION AND MATERIAL CHARACTERIZATION}

Commonly used piezoelectric films include PZT, ZnO, and AlN. [9-11]. In fabricating instrumented suspensions, the thin film fabrication process needs to be compatible with the steel substrate. The primary constraint is processing temperature due to the thermal expansion differential between steel and piezoelectric thin film materials. $\mathrm{ZnO}$ is a good choice because it can be deposited at a low temperature $\left(\sim 300^{\circ} \mathrm{C}\right)$ and is easier to deposit than PZT. Figure 4 illustrates the process flow for fabricating $\mathrm{ZnO}$ strain gages on steel suspensions. There were several special steps that were required to accommodate the steel substrate. The $40 \mu \mathrm{m}$ thick 304 stainless steel wafer has a rough surface from a microfabrication standpoint. Furthermore, it is flexible, conductive, subject to oxidation, and presents problems with thermal expansion differentials. First, a $0.7 \mu \mathrm{m}$ thick layer 


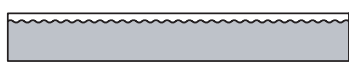

(a)

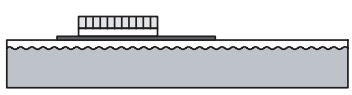

(b)

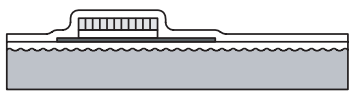

(c)

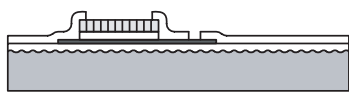

(d)

Spin-on-glass |||l|| Zinc oxide

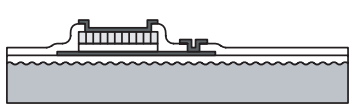

(e)

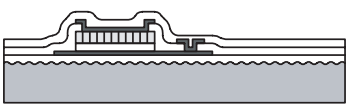

(f)

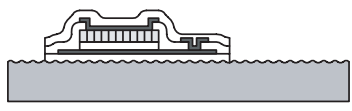

(g)

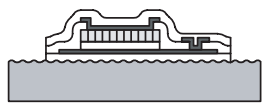

(h)

Steel substrate

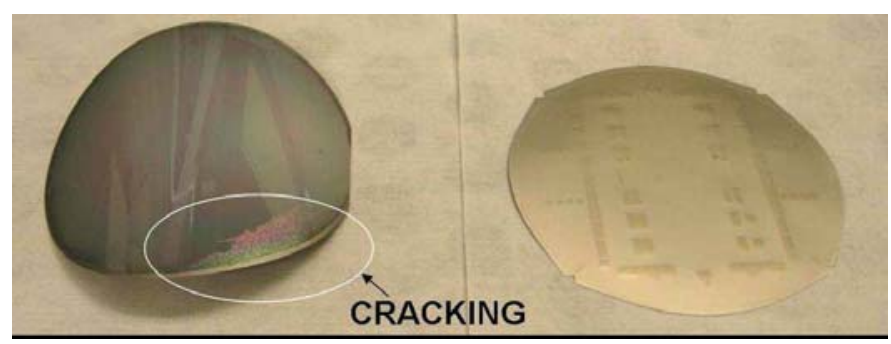

Figure 5. PROBLEMS WITH ZnO DEPOSITION ON STEEL WAFERS. THE WAFER ON THE LEFT HAD A BLANKET LAYER OF ZnO FILM ACROSS THE ENTIRE WAFER, RESULTING IN DRAMATIC WARPING AND CRACKING. THE WAFER ON THE RIGHT HAD PATCHES OF ZnO DEPOSITED THROUGH A STENCIL MASK, RESULTING IN LOCALIZED DIMPLES.

this method led to localized dimples, seen in Fig. 5, that rendered the subsequent lithography impossible. It was found that the most successful method was to constrain the wafer with a single bar across the center of the wafer, clamped to an underlying Si handle wafer. The bar divided the deposited area into two sections, somewhat reducing warping. After depositing the $\mathrm{ZnO}$, a small drop of water was carefully injected under the steel wafer to temporarily bond it to the Si handle wafer. Then the clamp was removed and the wafer remained flat for lithography. was deposited to aid with adhesion of the $\mathrm{ZnO}$ and to smooth out the stress gradient between the aluminum and $\mathrm{ZnO}$. A smooth surface was critical for subsequent $\mathrm{ZnO}$ deposition. The $\mathrm{ZnO}$ film was deposited using RF magnetron sputtering. The deposition rate at $300^{\circ} \mathrm{C}$ with $200 \mathrm{~W}$ forward power, $3.5 \mathrm{mTorr}$ oxygen, and 3.5 mTorr argon was approximately $0.8 \mu \mathrm{m}$ per hour. Films of $0.8-1 \mu \mathrm{m}$ have demonstrated good piezoelectric properties. The $\mathrm{ZnO}$ sensors were patterned using a wet etch consisting of a 1:10:100 ratio of phosphoric acid, acetic acid, and water, respectively. The underlying SOG layer was etched in $\mathrm{SF}_{6} / \mathrm{O}_{2}$ plasma using the $\mathrm{ZnO}$ as a mask. The bottom $\mathrm{Al}$ electrode and leads were then patterned and wet etched. A third SOG layer was deposited for insulation. Contacted holes were patterned in the SOG layer. A second layer of aluminum was evaporated and patterned for the top electrode and interconnects. One more layer of SOG was deposited for passivation and protection of the sensors. Finally, the steel wafers were bulk micromachined using a proprietary process to define the suspension geometry. At several steps throughout the process, it was necessary to bond the flexible wafers to Si handle wafers. An illustrated of a completed instrumented suspension is in Fig. 10.

The most challenging fabrication step posed by processing on a steel substrate was the sputtering, which introduces cracking and deformation due to residual stresses. When a single continuous film of $\mathrm{ZnO}$ was sputtered onto a steel substrate, the wafer became severely warped and widespread cracking was observed as seen in Fig. 5. In an attempt to address this problems, a stencil mask was incorporated into the process. $\mathrm{ZnO}$ was only deposited on the substrate where windows are cut out of the stencil mask. This drastically reduced overall wafer deformation. However,

\section{ZnO Film Characterization}

[12] describes in detail how $\mathrm{ZnO}$ sensors were fabricated on a simple Si cantilever for the purpose of estimating piezoelectric coefficients. The cantilever was mechanically excited with an impulse to generate high frequency vibration. The displacement at the tip of the cantilever was measured with a laser doppler velocimeter (LDV), while measuring the voltage signal from the sensors. Strain was related to displacement using fundamental beam theory for small deflections. The direct piezoelectric constant $e_{31}$ was then estimated from the calculated strain and measured sensor output voltage. Using this technique, the estimated direct piezoelectric constant was $e_{31}=.0377 \mathrm{C} / \mathrm{m}^{2}$. This was about an order of magnitude smaller than that reported in the literature $[13,14]$.

$\mathrm{X}$-ray diffraction (XRD) measurements were taken to further evaluate the $\mathrm{ZnO}$ material properties. Figure 6 shows the XRD spectrum of $\mathrm{ZnO}$ film deposited on a $\mathrm{Si}$ control wafer. The peak orientation measured at $34.4^{\circ}$ corresponds to the (002) plane. A rocking curve analysis determined the dispersion of this crystal plane from the desired orientation. A narrow dispersion indicates good piezoelectric film quality, as quantified by the full width at half maximum (FWHM) of the intensity from the rocking curve. A FWHM of $2.5^{\circ}$ was measured. FWHM of less than $2^{\circ}$ was desired based on previous results with the sputtering equipment used, but literature reports FWHM values for sputtered $\mathrm{ZnO}$ films between .9 and $12.47^{\circ}[15,16]$.

Because it was difficult to probe the patterned $\mathrm{ZnO}$ films on 


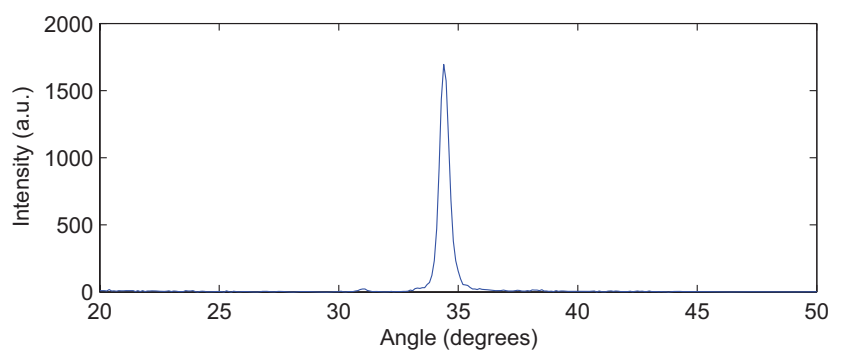

Figure 6. X-RAY DIFFRACTION SPECTRU FOR ZnO FILM DEPOSITED ON Si SUBSTRATE.

the steel wafer using XRD, piezoelectric film quality of the steel wafer and Si wafer were correlated using an experimental "tapper" apparatus. The tapper consisted of a tip probe that contains a PZT actuator. The sample was placed on a metal platter under the probe tip. The PZT actuator in the tip was excited by a sinusoidal voltage, generating a displacement in the material being tested. Charge was then generated across the tested material and measured as a voltage. Results tended to vary across the wafer and were dependent on the electrode material used on the sample. While this test was not suitable for extracting actual material properties, it was good for qualitatively comparing samples. The $\mathrm{ZnO}$ film on the Si control wafer generated between $40-80 \mathrm{mV}$, while the $\mathrm{ZnO}$ film on the steel process wafer generated between 60-120 $\mathrm{mV}$ for the same excitation amplitude. This indicates that the $\mathrm{ZnO}$ film on the steel wafer is comparable to the film fabricated on the Si control wafer that was used for XRD measurements.

Less than optimal $\mathrm{ZnO}$ film quality was attributed to several factors. First, the sputtering system used to deposit the films was not reaching an optimally low base pressure. In addition, parasitic capacitance in the experimental set up reduced the measured piezoelectric constant. However, as will be seen in the following sections, the piezoelectric response was adequate for sensing vibrations.

\section{EXPERIMENTAL TESTING \\ Interface Circuit}

Due to both material properties and size scale, the $\mathrm{ZnO}$ sensors produced a very small charge in response to external strain, on the order of picoamps. Therefore, an appropriate interface circuit was required to condition and amplify the signal. It was also critical to place the circuit close to the sensors to minimize environmental noise and feed-through. Figure 8 illustrates a diagram of the circuit. It was designed to amplify signals in the frequency range of $1 \mathrm{kHz}$ and $30 \mathrm{kHz}$ where the vibrational modes of interest are found. The circuit was composed of two stages, a buffer stage and a gain stage. The buffer stage utilized a differential input to reduce the common mode noise from both leads with gain of 100. The relatively large gain was desirable to amplify the signal and increase signal-to-noise ratio (SNR). The second

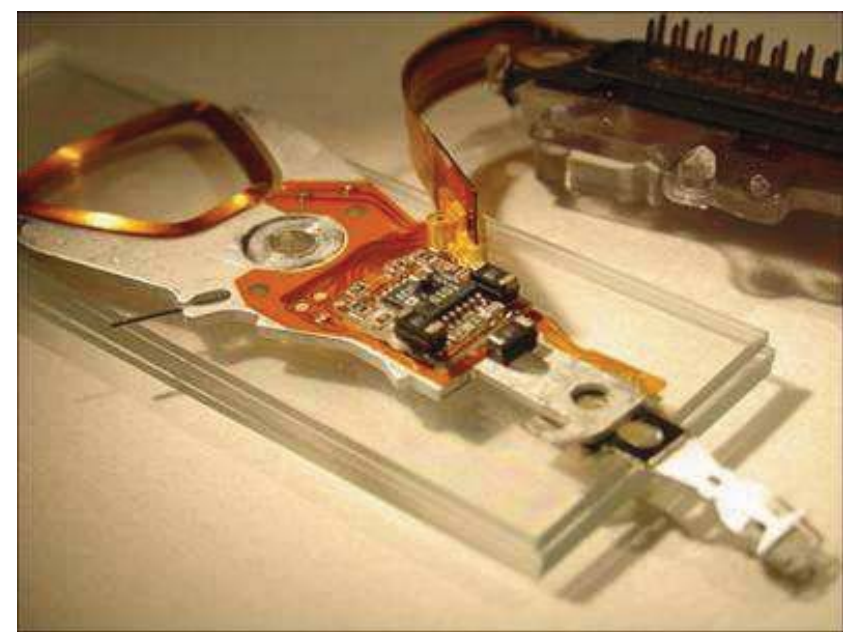

Figure 7. THE E-BLOCK AND SUSPENSION ASSEMBLY WITH INTERFACE CIRCUIT ATTACHED.

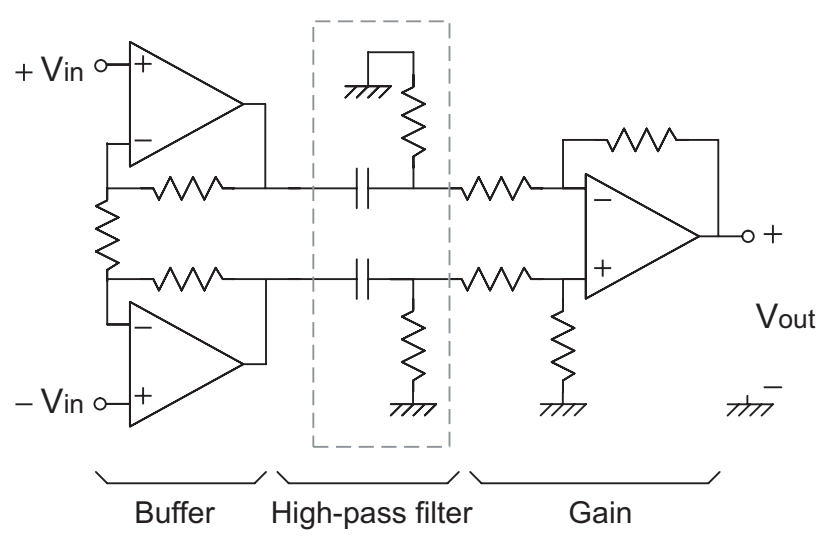

Figure 8. SCHEMATIC OF INTERFACE CIRCUIT.

stage was a differential-to-single-end converter with gain of 10 . A high pass filter was added between the two stages to prevent low frequency noise and drift from being amplified.

\section{Experimental Setup}

Due to the limited space inside hard disk drives, surface mount components were used to build the interface circuit directly onto the E-block. Figure 7 shows the circuit installed on a hard drive E-block. The drive's flex circuit was utilized to pass signals from the sensors to the circuit and from the circuit to pins at the back side of the drive. The instrumented suspension prototypes used in this experiment were designed to be installed with a $3 \mathrm{~mm} \times 3 \mathrm{~mm}$ electrostatic microactuator [6]. However, the purpose of this test was to prove out the use of the sensors, so a functioning microactuator was not used. To simulate the mass of the actuator and obtain the correct flying height, immobilized dummy microactuators were installed on the gimbal of the exper- 


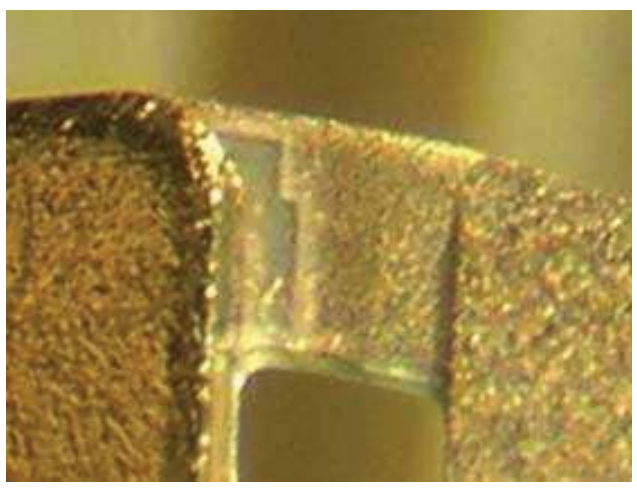

Figure 9. PHOTOGRAPH OF ACTUAL SENSORS NEAR THE HINGE OF A SUSPENSION PROTOTYPE.

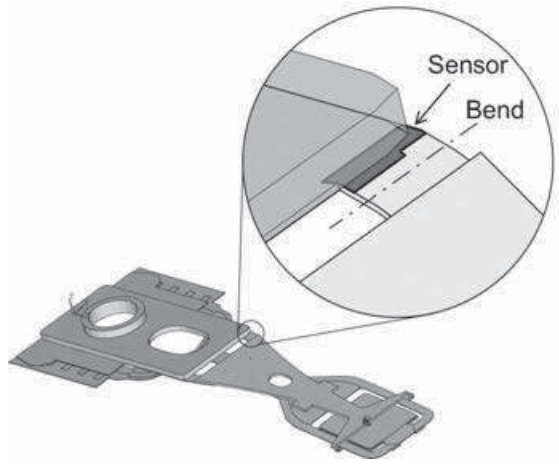

Figure 10. AN ILLUSTRATION OF A SENSOR ON THE SUSPENSION, DESIGNED USING THE OPTIMIZATION METHOD DISCUSSED IN THIS PAPER.

imental suspensions. Figure 9 shows a close view of the hinge area on the suspension where sensors are located. The sensors were about $.1 \mathrm{~mm}^{2}$ and about $4 \mu \mathrm{m}$ in total thickness. Note that on these prototypes, the two sensors were not interconnected for mode cancelation. A LDV was used to measure displacement.

\section{Results}

Several measurements were taken in order to compare the LDV displacement response to the sensor response. For ease of measurement, these tests were done without a disk installed and the spindle off. An HP35665A analyzer was used to obtain transfer functions from VCM input to sensor output and from VCM input to LDV output. Figure 11, top, shows the the sensor response. Figure 11, middle and bottom, show the response from off-track and non-off-track LDV measurements, respectively. The sensor response contained the same suspension modes that appear in the off-track LDV measurement. Additional modes were resolved by the sensors at higher frequencies, indicating better SNR than the LDV measurement. The sensors also picked up some non-off-track modes, as expected.

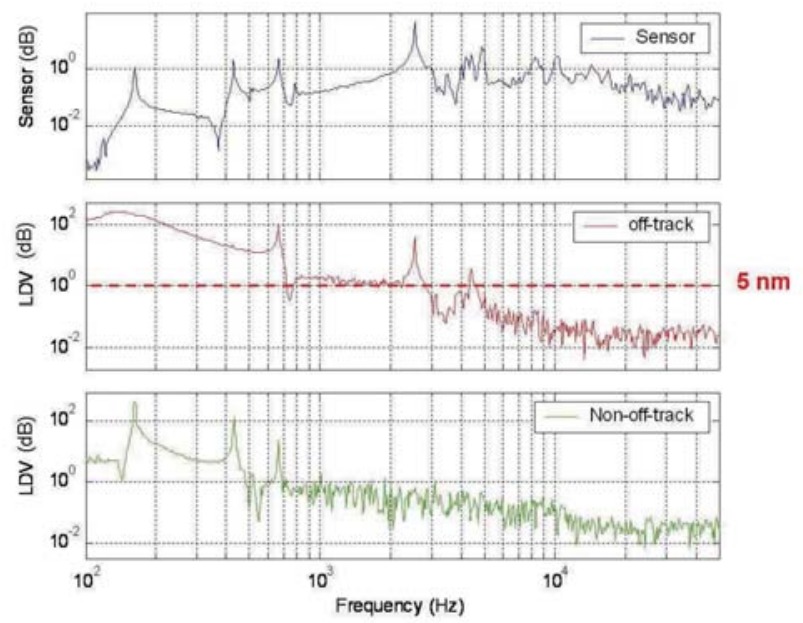

Figure 11. TRANSFER FUNCTION ON STAND-ALONE SUSPENSION, FROM VCM TO AMPLIFIED SENSOR SIGNAL (TOP), FROM VCM TO OFF-TRACK LDV MEASUREMENT (MIDDLE), AND FROM VCM TO NON-OFF-TRACK LDV MEASUREMENTS (BOTTOM).

Next, open loop transfer functions were obtained with the slider flying on a spinning disk. Off-track displacement was again obtained using an LDV. These transfer functions are shown in Fig. 12. Once again, the sensor detected the same modes that appear in the LDV off-track displacement measurement. In addition, it resolved higher frequency modes, and picked up some lower frequency modes that were attributed to non-off-track displacement. Note that the modes that appeared are not the same as in the test results without a disk. When the suspension is flying on a disk, the air bearing tends to stiffen the structure, shifting the resonance modes to higher frequencies. Also, the experimental drive had to be rebuilt between tests, introducing some variation in the assembly. Finally, somewhat higher noise was encountered during this test, resulting in a less clean sensor signal. Based on the periodic nature of the noise observed, it was possible that some of this noise was an environmental disturbance.

\section{MODELING AND CLOSED LOOP SIMULATIONS}

Simple closed-loop control simulations were performed to evaluate the effectiveness of strain sensing in hard drive tracking control, using only VCM actuation. This section describes the model assumptions, control design and simulation results.

\section{Model}

Modal dynamics were identified from the experimental data obtained in the previous section with the disk spinning. A number of modes were neglected in the interest of keeping the order of the controller manageable. Also, in the model of the sensor response, non-off-track modes are ignored, since it is as- 

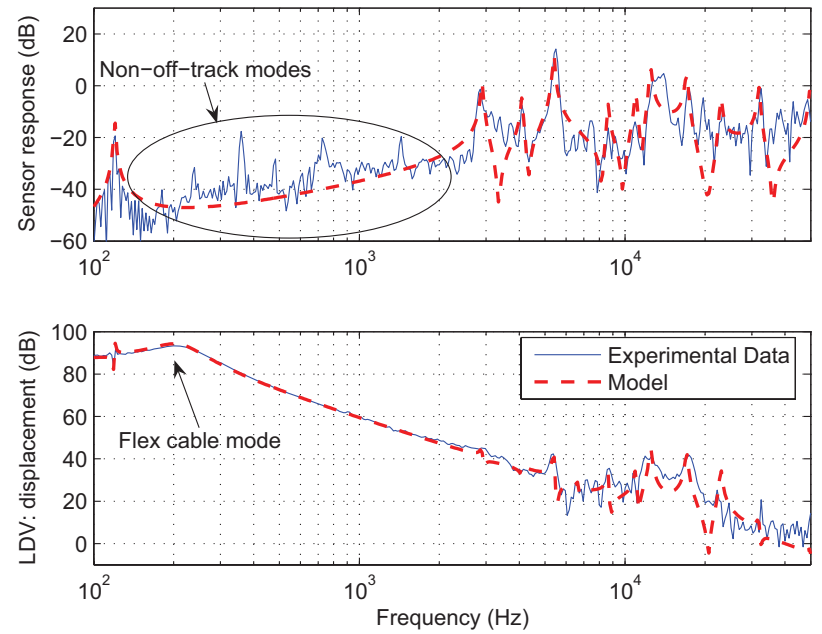

Figure 12. TRANSFER FUNCTIONS WITH DISK SPINNING, FROM VCM TO AMPLIFIED SENSOR SIGNAL (TOP), AND FROM VCM TO OFF-TRACK LDV MEASUREMENT (BOTTOM).

sumed that symmetrical, interconnected sensors can cancel these modes. The magnitude responses of the models are shown in Fig. 12. While suspension modes appeared in both the sensor and LDV measurements, these two responses have different underlying dynamics. The interface circuit dynamics were included in the transfer function from the VCM to the strain sensors, while a flex cable mode was included in the transfer function from the VCM to the head displacement.

The system is subject to several exogenous input disturbances. Track runout, which consists of low frequency repeatable and non-repeatable disturbances, was modeled as white noise, $r$, filtered by a transfer function, $G_{R}(s) . G_{R}(s)$ was obtained from [17] and is shown in Fig. 13. Windage disturbance was also modeled as a white noise input with a scaled excitation of each mode, normalized to produce an RMS value $6 \mathrm{~nm}$ head displacement. The windage model is also shown in Fig. 13. Measurement noise was estimated to be $.1 \mathrm{~V}$ from the signal observed while extracting the experimental data shown in Fig. 12. The PES noise was assumed to be $1 \mathrm{~nm}$, as in [17]. The PES sample rate was $50 \mathrm{kHz}$.

\section{Closed-Loop Simulation}

For the purpose of design evaluation, an $\mathrm{H}_{2}$ controller was synthesized based on the nominal model, which minimizes the variance of the PES subject to the stochastic disturbances described in the previous section. The input disturbances (windage and runout) were considered one at a time, to compare how well the sensing handles each. Tracking performance was quantified by the RMS value of the head position error. A weighting factor on the control input was selected to constrain the VCM voltage to an RMS value of $1.67 \mathrm{~V}$.

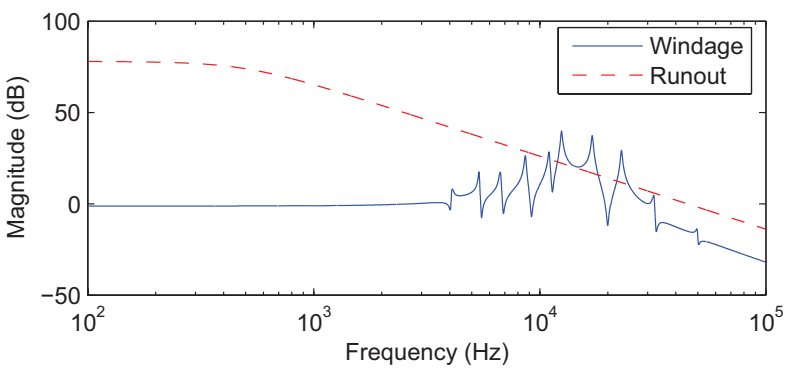

Figure 13. EXOGENOUS INPUT DISTURBANCE MODELS.

Table 1. H2 SIMULATION RESULTS.

\begin{tabular}{lll}
\hline Sensing & $\begin{array}{l}\text { RMS Position } \\
\text { Error }(\mathrm{nm})\end{array}$ & $\begin{array}{l}\text { Percentage At- } \\
\text { tenuation }\end{array}$ \\
\hline Open Loop & 6.3 & \\
PES, 50kHZ & 2.7 & $57 \%$ \\
PES and $y_{S}, 50 \mathrm{kHz}$ & 2.6 & $59 \%$ \\
$y_{S}$ only, $50 \mathrm{kHz}$ & 3.6 & $42 \%$ \\
$y_{S}$ only, $100 \mathrm{kHz}$ & 2.5 & $60 \%$ \\
\hline \hline
\end{tabular}

The open loop RMS head position in the face of windage was $6.3 \mathrm{~nm}$. Using only the PES for vibration suppression, this error was reduced to $2.7 \mathrm{~nm}$. By adding strain sensing at the same rate, the error was further reduced to $2.6 \mathrm{~nm}$, likely due to the improved resolution. Using only strain sensing at $50 \mathrm{kHz}$, the RMS head position error was reduced to $3.6 \mathrm{~nm}$. This is likely because the strain sensors do not detect displacement from the flex cable mode. However, if the strain sensor was used alone at a rate of $100 \mathrm{kHz}$, error was reduced to $2.5 \mathrm{~nm}$. The results of the simulated cases with windage disturbance are shown in Tab. 1. In the case of track runout disturbance, the PES alone reduced the RMS tracking error from $450 \mathrm{~nm}$ in open loop to about 17 $\mathrm{nm}$. Adding strain sensing at the same rate resulted in the same closed loop RMS value of $17 \mathrm{~nm}$. To summarize the results:

1. Adding strain sensing to the PES at a single rate improved tracking compared to sensing with the PES alone.

2. Vibration suppression from strain sensing improved significantly at a higher rate.

3. Strain sensing did not improve attenuation of track runout disturbance when sampled at the same rate as the PES.

The values for the closed-loop PES were heavily dependent on the disturbance models assumed. Also, nominal $H_{2}$ control is not robust and therefore unsuitable for implementation. A robust controller would sacrifice some tracking performance for stability. With this in mind, the relative improvement in PES RMS was of primary interest in this simulation study. 


\section{CONCLUSION AND FUTURE WORK}

This paper described the integration of thin film $\mathrm{ZnO}$ strain sensors onto hard disk drive suspensions for improved vibration suppression and tracking control. Sensor location was designed using an efficient optimization algorithm based on linear quadratic gaussian (LQG) control, which weighted modes that contribute to off-track error. Deposition of $\mathrm{ZnO}$ films was successfully adapted to steel substrates that were subsequently fabricated into instrumented suspensions. Prototype instrumented suspensions were installed and tested in an operating commercial hard drive. The strain sensing signal demonstrated superior SNR compared to LDV measurements. Preliminary models were identified from experimental data, including input-output transfer functions and measurement noise. Closed-loop simulations using nominal $\mathrm{H}_{2}$ control demonstrated improved vibration suppression as a result of both the better resolution and higher sensing rate provided by the sensors.

The closed-loop analysis in this paper was useful for design validation, but somewhat impractical for implementation. First, nominal $\mathrm{H}_{2}$ control was used, which is not robust. Secondly, only single rate control was used. A real servo system with strain sensing would be a multi-rate system requiring more elaborate control design. Finally, it was assumed that non-offtrack modes appearing in the strain signal could simply be canceled using symmetry. This was optimistic, since non-off-track torsional modes cannot be canceled in this way and variations in the two sensors would prevent perfect cancelation.

Future work will focus on formulating appropriate control designs that make use of additional strain sensing. Such control strategies will include multi-variable, multi-rate robust control synthesis, and adaptive feed forward control. Adaptive feed forward control could address issues of unknown disturbances and variations in sensor response. Dual-stage actuation is another promising strategy to achieve better tracking precision. We are currently fabricating a second-generation instrumented suspension prototype that will incorporate PZT actuators. These prototypes will be used to demonstrate the combined effectiveness of dual-stage actuation and high-rate strain sensing in achieving tracking performance and thus high data densities.

\section{ACKNOWLEDGMENT}

This work was supported by the National Science Foundation grant CMS-0428917, the Information Storage Industry Consortium, and the Computer Mechanics Laboratory at U.C. Berkeley. We also collaborated closely with Hutchinson Technology.

\section{REFERENCES}

[1] Evans, R., Griesbach, J., and Messner, W., 1999. "Piezoelectric microactuator for dual-stage control". IEEE Trans. Mag., 35, March, pp. 977-82.

[2] Hirano, T., Fan, L., Semba, T., Lee, W., Hong, J., Pattanaik, S., Webb, P., Juan, W.-H., and Chan, S., 1999. "High- bandwidth hdd tracking servo by a moving-slider microactuator". IEEE Trans. Mag., 35, September, pp. 3670-72.

[3] Fujita, H., Suzuki, K., Ataka, M., and Nakamura, S., 1999. "A microactuator for head positioning system of hard disk drives". IEEE Trans. Mag., 35, March, pp. 1006-10.

[4] Li, Y., Horowitz, R., and Evans, R., 2003. "Vibration control of a pzt actuated suspension dual-stage servo system using a pzt sensor". IEEE Trans. Mag., 39, March, pp. 9327.

[5] Kondoh, C., Inoue, S., and K., I., 1990. "The positioning of sensors and actuators in the vibration of flexible systems". JSME Intl. J., 33, pp. 145-152.

[6] Oldham, K., Kon, S., and Horowitz, R., 2004. "Fabrication and optimal stain sensor placement in an instrumented disk drive suspension for vibration suppression". In Proc. of the 2004 American Control Conference, pp. 1855-1861.

[7] Kon, S., Oldham, K., Ruzicka, R., and Horowitz, R., 2006. "Design and fabrication of a piezoelectric instrumented suspension for hard disk drives". In Proc. of SPIE, Vol. 6174, pp. 617430.1-.10.

[8] Antsaklis, P., and Michel, A., 1998. Linear Systems. McGraw-Hill.

[9] Hsua, Y.-C., Wub, C.-C., Leeb, C.-C., Caoc, G. Z., and Shen, I. Y., 2005. "Demonstration and characterization of pzt thin-film sensors and actuators for meso- and microstructures". Thin Solid Films, 485, pp. 42-46.

[10] DeVoe, D., 2001. "Piezoelectric thin film micromechanical beam resonators". Sensors and Actuators A: Physical, 88, pp. 263-272.

[11] Piazza, G., and Pisano, A., 2007. "Two-port stacked piezoelectric aluminum nitride contour-mode resonant mems". Sensors and Actuators A: Physical, 136, pp. 638-645.

[12] Kon, S., 2007. "High resolution mems strain sensors for vibration detection on hard disk drive instrumented suspensions". Phd thesis, University of California, Berkeley, May.

[13] Nalwa, H. S. Handbook of Thin Film Materials, Vol. 3. Academic Press.

[14] Carlotti, G., Socino, G., Petri, A., and Verona, E., 1987. "Acoustic investigation of the elastic propertied of zno films". Applied Physics Letters, 51, pp. 1889-1891.

[15] Perluzzo, G., Jen, C., and Adler, E., 1989. “Characteristics of reactive magnetron sputtered zno films". In Proc. of the Ultrasonics Symposium, Vol. 1, pp. 373-376.

[16] Lee, J., Sanghyon Kwack, S., and Kim, H. J., 2001. "Effects of substrates roughness on c-axis preferred orientation of zno films deposited by rf magnetron sputtering”. In Material Research Society Symposium Proc., Vol. 672, pp. O8.20.1-O8.20.6.

[17] Huang, X., Nagamune, R., and Horowitz, R., 2006. “A comparison of multirate robust track-following control synthesis techniques for dual-stage and multi-sensing servo systems in hard disk drives". IEEE Trans. Mag., 42, July, pp. 1896-904. 